\title{
How substantial are ultraviolet-B supplementation inaccuracies in experimental square-wave delivery systems?
}

\author{
C.F. Musil ${ }^{a}$, L.O. Björn ${ }^{\text {b }}$, M.W.J. Scourfield ${ }^{c}$, G.E. Bodeker ${ }^{d}$ \\ ${ }^{a}$ Research Scientific Services, National Botanical Institute, Private Bag X7, Claremont, 7735 Cape Town, \\ South Africa \\ Department of Plant Physiology, Lund University, Box 117, S -221 00 Lund, Sweden \\ School of Pure and Applied Physics, University Natal, Durban 4041, South Africa \\ d National Institute Water and Atmospheric Research, Lauder, Central Otago, New Zealand \\ *Corresponding author. Tel.: + 27-21-799-8710; fax: + 27-21-797-6903. \\ E-mail address: musil@nbict.nbi.ac.za
}

\begin{abstract}
Square-wave (SQW) ultraviolet-B (UV-B: 280-315 nm) radiation supplementation systems continue to be used in outdoor experimental locations due to the economically restrictive installation and maintenance costs, and technical expertise required to effectively operate more advanced modulated (MOD) delivery systems. However, continued yet contentious criticisms of SQW delivery systems risk creating prejudices as to the validity of plant responses measured in these with potentially negative repercussions on future UV-B experimentation. Consequently, we quantified the magnitude of UV-B supplementation inaccuracies in our typical outdoor step-wise SQW delivery system using 7-year records of computer-modeled and instrument-measured solar UV-B irradiances and synchronous measurements of total solar (300-3000 nm) radiation and daily sunshine duration. Both broad-and narrow-band instrument measurements confirmed that our step-wise SQW delivery system rendered larger total daily supplemental UV-B exposures (time-integrated UV-B irradiances) than a MOD delivery system on only substantially overcast days ( $25 \%$ or less daily sunshine duration). These larger supplemental UV-B exposures were augmented with increased magnitude of the added artificial UV-B supplement. However, their ranges did not exceed those in a MOD delivery system by more than $10 \%$ for added UV-B supplements of realistic magnitude ( $30 \%$ or less above background), except on virtually completely overcast days ( $5 \%$ or less daily sunshine duration). Also, our step-wise SQW delivery system rendered higher photon flux ratios of UV-B/total solar radiation than a MOD delivery system on only substantially overcast days, the ranges of which were also augmented with increased magnitude of the added artificial UV-B supplement. However, these features were restricted to high solar angles, since with reduced solar angle these higher photon flux ratios also included progressively less overcast days. Nevertheless, ranges of photon flux ratio increases were well below reported thresholds inhibiting to plant growth at all solar angles for the added artificial UV-B supplements of realistic magnitude, except on virtually completely overcast days. Results point to an under-estimation of clear-sky UV$\mathrm{B}$ irradiance by the computer-encoded semi-empirical model commonly utilized to predict background and supplemental UV-B irradiances for SQW delivery systems. They confirm the superiority of MOD delivery systems in providing more realistic conditions of UV-B increases but likewise demonstrate little justification on results derived from all field-based SQW delivery systems as exaggerated where sensible irradiation protocols and realistic UV-B supplements are applied.
\end{abstract}


Keywords: Daily sunshine duration; Modulated delivery; Photon flux ratio; Solar radiation; Ultraviolet-B radiation

\section{Introduction}

Ultraviolet-B (UV-B: $280-315 \mathrm{~nm}$ ) radiation, though representing only a small fraction of the total solar electromagnetic spectrum, exerts a disproportionately large photobiological effect on living organisms due to its absorption by important biological macromolecules, such as proteins and nucleic acids (Jansen et al., 1998). Stratospheric ozone is an important attenuator of solar UV-B radiation (Rozema et al., 1999). Reports of its decline globally, linked to the industrial emissions of chlorofluorocarbons (Fraser and Prather, 1999), has prompted research into the effects of solar UV-B increases on agricultural and natural plant ecosystems (Caldwell et al., 1995; Rozema et al., 1997; Laakso and Huttunen, 1998).

Early research conducted predominantly in growth-chamber and glasshouse environments did not adequately simulate the overall radiation environment (Teramura, 1983). Subsequent outdoor studies have utilized artificial sun lamps to supplement background solar UV-B radiation (McLeod, 1997). However, lamp spectral characteristics do not precisely match the change in solar spectrum caused by ozone depletion (Caldwell et al., 1986). Because of this feature, and the strong dependency of photon absorption on wavelength, weighting functions based on action spectra for specific responses have been advanced to assess the biological effectiveness of both the UV-B irradiation source and predicted ozone depletion (Rundel, 1983). Uncertainties surrounding weighting functions include their specificity to a particular biological response, and limitation to the experimental conditions used to generate the action spectrum (Coohill, 1991, 1992). Indeed, most analytical action spectra have been generated from biological responses to monochromatic radiation in the laboratory over hours. Ideally, spectral responses over extended periods of days, weeks and months to the full solar spectrum in the field are required to accurately evaluate the relevance of biological responses to ozone depletion (Flint and Caldwell, 1996).

Apart from these and other qualitative issues, e.g. optical stability of filters used to create UV-B treatments and controls (Middleton and Teramura, 1993; Döhring et al., 1996; Newsham et al., 1996), there are more immediate concerns regarding the quantitative delivery of desired UV-B radiation in outdoor supplementation systems. The great majority of field experiments have used square-wave (SQW) delivery systems, in which lamps provide constant UV-B supplements representing a chosen ozone depletion scenario for a particular day of the year under assumed clear-sky conditions. Lamp supplements in such delivery systems are often supplied in a step-wise manner to compensate partly for diurnal changes in solar zenith angle, and also adjusted for seasonal variations in UV-B radiation. Spectral models provide the required UV-B radiation supplements for specified geographic locations, times of the year and ozone thickness. A variety of spectral models covering a multitude of approaches ranging various approximations of the radiative transfer equation to semi-empirical models have been advanced (Nunez et al., 1994). One developed by Green and co-workers (Green et al., 
$1974,1980)$ has the capacity for interpolation and extrapolation to other sets of environmental conditions (Rundel, 1986). This, with algorithmic revisions (Green, 1983) has been incorporated into a readily accessible computer program (Björn and Murphy, 1985) frequently used by biologists to predict background solar UV-B radiation levels, and required experimental UV-B supplements to simulate a given stratospheric ozone depletion scenario on both instantaneous and daily bases.

Modulated (MOD) field irradiation systems have also been developed and employed by a few laboratories in which UV-B supplements are supplied as proportional increments to constantly monitored background UV-B conditions (Caldwell et al., 1983; McLeod, 1997). These delivery systems account for diurnal, seasonal, and cli-materelated fluctuations in solar UV-B radiation by maintaining a constant supplemental to background UV-B ratio, and place relatively less emphasis on computer-modeled predictions of UV-B irradiance, since background UV-B intensity continuously controls the incremental UV-B supplement. As a consequence, SQW delivery systems have come under increased criticism (Sullivan et al., 1994; Allen et al., 1998). The rationale advanced include semi-empirical model over-estima-tion of the supplementary UV-B irradiance for a given ozone depletion scenario, and absence of supplemental UV-B adjustments for local changes in climate, such as variations in the amount and form of cloud and atmospheric aerosols. These has been argued, invariably lead not only to greater UV-B supplementation than intended but also to substantially higher total photon flux ratios of UV-B to UV-A, and photosynthetically active radiation (PAR) than would naturally occur in response to ozone depletion (Fiscus and Booker, 1995). These ratios are particularly important in determining how sensitively plants respond to changes in UV-B (Smith et al., 1992; Caldwell et al., 1994).

Alleged UV-B supplementation excesses in SQW delivery systems, though seemingly valid from a theoretical perspective are controversial. One 5-month comparison between a SQW and MOD delivery system indicated that the daily average total UV-B irradiance received by plants was up to $30 \%$ greater in the SQW delivery system (Sullivan et al., 1994). Mere down regulation of the system to compensate for this increase was problematic due to difficulties in forecasting constantly changing weather conditions necessary to adjust SQW systems to give the same daily exposures as MOD systems resulting in either over-or under-exposure. However, it was also acknowledged that these estimates, which were based on an admixture of spectroradiometer, broad-band UVdetector and pyranometer converted measurements, probably represented a worst-case scenario. Notable in this regard also are conflicting reports on semi-empirical model precision in predicting UV-B irradiance. Over-estimation of ground level UV-B irradiance, especially at lower solar angles, has been demonstrated for earlier versions (Green et al., 1974, 1980) of the semi-em-pirical model (Fiscus and Booker, 1995). However, later revisions (Green, 1983), computer-encoded by Björn and Murphy (1985), show model deviations from spectroradiometer-mea-sured UV-B irradiance spectra ranging from 0 to $20 \%$ at three different latitudes, though these did exceed a factor of 3 at $300 \mathrm{~nm}$ at one location (Björn and Murphy, 1985). Another independent study also demonstrated relatively close correspondence between erythemal-weighted UV-B irradiances predicted with the Björn and Murphy (1985) computer-encoded semi-empirical 
model and those measured with a broad-band (Robertson-Berger) meter (Booker et al., 1992).

SQW UV-B supplementation systems continue to be used in outdoor experimental locations due to the economically restrictive installation and maintenance costs, and technical expertise required to effectively operate more advanced MOD delivery systems. However, continued yet contentious criticisms of SQW delivery systems risk creating prejudices as to the validity of plant responses measured in these with potentially negative repercussions on future UV-B experimentation. Therefore, it is imperative that the alleged UV-B delivery inaccuracies associated with SQW supplementation systems are properly quantified so that realistic judgements of their magnitude can be made and corrective actions considered.

\section{Methods}

2.1. Description of monitoring site

The monitoring site was the University of Natal situated in the industrialized coastal city of Durban $\left(29^{\circ} 58 \% \mathrm{~S} ; 30^{\circ} 57 \% \mathrm{E}\right)$. The site's subtropical climate is warm and humid, with a mean annual rainfall of $1018 \mathrm{~mm}$ (Climate of South Africa, 1986) averaging 26 $\mathrm{mm}$ precipitation and 28\% cloud cover during June (midwinter) and $135 \mathrm{~mm}$ precipitation and $65 \%$ cloud cover during January (midsummer).

\subsection{Modeled solar ultraviolet- $B$ radiation}

A freely available computer-encoded semi-em-pirical model (Björn and Murphy, 1985) was used to predict clear-sky solar UV-B irradiances in the $280-315 \mathrm{~nm}$ range. A few subroutines were added to this program. These allowed the following:

1. Input of authentic total column ozone values into the program.

2. Automated computation at 10-min intervals for southern hemisphere locations of absolute UV-B irradiances weighted by the generalized plant response action spectrum (Caldwell, 1971) as mathematically formulated by Green et al. (1974), and normalized at $300 \mathrm{~nm}$.

3. Time integration of all UV-B irradiance computations into total daily UV-B exposures using the following formula:

$$
\text { Total daily UV-B exposure }\left(\mathrm{kJ} \mathrm{m}^{-2} \mathrm{~d}^{-1}\right)=0.06\left[\sum 0.5\left(\left(\mathrm{t}_{2}-\mathrm{t}_{1}\right)\left(\operatorname{Ir}_{2}+\mathrm{Ir}_{1}\right)\right)\right]
$$

are $t_{2}-t_{1}$ were the times ( $\min$ ) between consecutive measuring intervals, and $\operatorname{Ir}_{1}$ and $\operatorname{Ir}_{2}$ were the predicted plant-effective UV-B irradiances $\left(\mathrm{W} \mathrm{m}^{-2}\right)$ at the beginning and end of each measuring interval.

Total column ozone values imputed into the program were obtained from NASA TOMS instrumentation flown on board the Nimbus 7 (McPeters et al., 1996), Meteor (Herman et al., 1996), Earth Probe (McPeters et al., 1998) and Adeos (Krueger et al., 1998) satellites, and also the Global Ozone Monitoring Experiment (GOME) flown on the ERS-2 satellite (Burrows et al., 1999). Ozone values closest to the Durban location were extracted from $1^{\circ}$ latitude by $1.25^{\circ}$ longitude satellite grid data using bilinear interpolation. Due to the differential satellite orbits, sampling close to local midday was 
every $24 \mathrm{~h}$ for TOMS and every $72 \mathrm{~h}$ for GOME. UV-B irradiance computations used standard program defaults for an urban environment and ground cover corresponding with green farmland, which best described the monitoring site surrounds. Barometric pressure, atmospheric relative humidity and aerosol level inputs into the program were standardized at $950 \mathrm{mbar}, 50 \%$ and 1.0, respectively. The modeled solar UV-B irradiances, with associated times and solar zenith angles, and time-integrated total daily UV-B exposures covered the 2618-day period from March 1993 to April 2000 with intermittent gaps (about 935 days in total) due to incomplete satellite ozone coverage.

\subsection{Measured solar ultraviolet- $B$ radiation}

Measurements of solar UV-B radiation were obtained concurrently with broad-and narrowband instruments located in an observation tower, which allowed free view to within a few degrees of the true horizon. The broad-band instrument comprised an internally temperature-stabilized pyranometer (YES model UV-B1, Yankee Environmental Systems, Inc. Turners Falls, MA), which records incident UV-B irradiance every $15 \mathrm{~s}$ over $2 \pi \mathrm{sr}$, i.e. with full view of the observable horizon. The instrument's analog voltage output, with a cosine response of less than $5 \%$ for $0-60^{\circ}$ solar zenith angle, was interfaced with a data logger, which digitized and averaged readings over 10min intervals. The averaged readings were downloaded daily to a personal computer, which computed solar zenith angles, converted analog voltage readings into absolute and biologically weighted irradiances compliant with the generalized plant action spectrum, and time-integrated all UV-B irradiance measurements into total daily UV-B exposures. The solar UV-B irradiance measurements with associated times and solar zenith angles, and time-integrated total daily UV-B exposures covered the 2618-day period from March 1993 to April 2000 with intermittent gaps (about 455 days in total) due to instrument performance and calibration checks.

The narrow-band instrument comprised a double monochromator spectroradiometer (Bentham model DM 150, Bentham Instruments Ltd. Reading, Berkshire, UK) interfaced with a personal computer. The light sensor was a fiber optic cable, topped by a teflon diffuser, connected to a temperature-stabilized photomultiplier detector sealed in an insulated container. The instrument was calibrated monthly for absolute response against an incandescent lamp (Bentham CL2, Bentham Instruments Ltd.) mounted inside a baffled cylinder to exclude ambient and reflected lamp light, and simultaneously checked for wavelength alignment using vapor emission lines from a mercury arc lamp. Scans at 2 $\mathrm{nm}$ intervals between 280 and $450 \mathrm{~nm}$ were taken daily for every $5^{\circ}$ of solar zenith angle. The spectral irradiance measurements were biologically weighted with the generalized plant action spectrum, and all weighted UV-B measurements in the range $280-315 \mathrm{~nm}$ time-integrated into total daily UV-B exposures. The UV-B irradiance measurements, with associated times and solar zenith angles, and time-integrated total daily UV-B exposures covered the 1277-day period from November 1996 to April 2000 with intermittent gaps (about 173 days in total) due to instrument performance and calibration checks.

\subsection{Measurements of total solar radiation and sunshine duration}

Digitized total solar (300-3000 nm) radiation measurements averaged hourly, and 
parallel sunshine duration measurements covering the period from March 1993 to April 2000 were supplied by the South African Weather Bureau for a proximal meteorological station at the Durban International Airport. Measurements of total solar radiation (TSR) were obtained from a Moll-Goranski thermopile housed in a Kipp and Zonen CM5 pyranometer (Delft, The Netherlands). Parallel sunshine duration measurements were obtained with a Campbell-Stokes type recorder and expressed as a fraction of total day length. These were considered a more reliable correlate of UV attenuation than cloud amount, which exhibits a poor functional dependency on TSR. By implication, this should also apply to biologically weighted UV-B irradiances, since there are several reports of similar attenuation by cloud of TSR and UV-B irradiances weighted with the McKinley and Diffey (1987) reference erythemal action spectrum (Frederick and Steele, 1995; Bodeker and McKenzie, 1996; Thiel et al., 1997). More recent assignment of UVirradiation data to distinct cloud forms has yielded satisfactory cubic relationships (Thiel et al., 1997), but their application was an impractical proposition in this study due to the general paucity of cloud form records in the meteorological archives.

\subsection{Comparison of square-wave and modulated delivery systems}

\subsubsection{Total daily supplemental ultraviolet-B exposures}

Artificial UV-B supplements of different magnitude (10, 20 and 30\% above background) were added to broad-and narrow-band instrument measurements of UV-B irradiance in accordance with SQW and MOD delivery principles. In the SQW delivery system, the artificial UV-B supplements added to the instrument measured background UV-B irradiances were computed from a semi-empirical model and spread over an 8-h artificial irradiation period centered on the solar noon (12:00 h South African Standard Time at $30^{\circ} \mathrm{E}$ ). A step-wise irradiation protocol was adopted. Two-thirds of the modeled UV-B supplement was spread over the 4-h photoperiod centered on the solar noon, with the remaining one-third spread equally over the two extra $2-\mathrm{h}$ photoperiods at either ends of the day. In practice, these diurnal UV-B intensity adjustments were achieved by switching-on greater numbers of lamps in the banks during the solar noon centered photoperiod than that at either ends of the day. Seasonal UV-B intensity adjustments were achieved by varying the distance between the lamps and plant apices. In the MOD delivery system, the added artificial UV-B supplements were merely proportional increments $(10,20$ or $30 \%$ above background) of the constantly instrument-monitored ambient UV-B irradiance. Illustrated step-wise SQW and MOD delivered supplemental UV-B exposures for completely and slightly overcast days are presented in Fig. 1(A) and (B). For example, measured background UV-B irradiances on the completely overcast Julian day 7 of 1998 (sunshine duration $0.7 \%$ of day length) integrated over time gave a total daily UV-B exposure of $4.286 \mathrm{~kJ} \mathrm{~m}^{-2} \mathrm{~d}^{-1}$. 

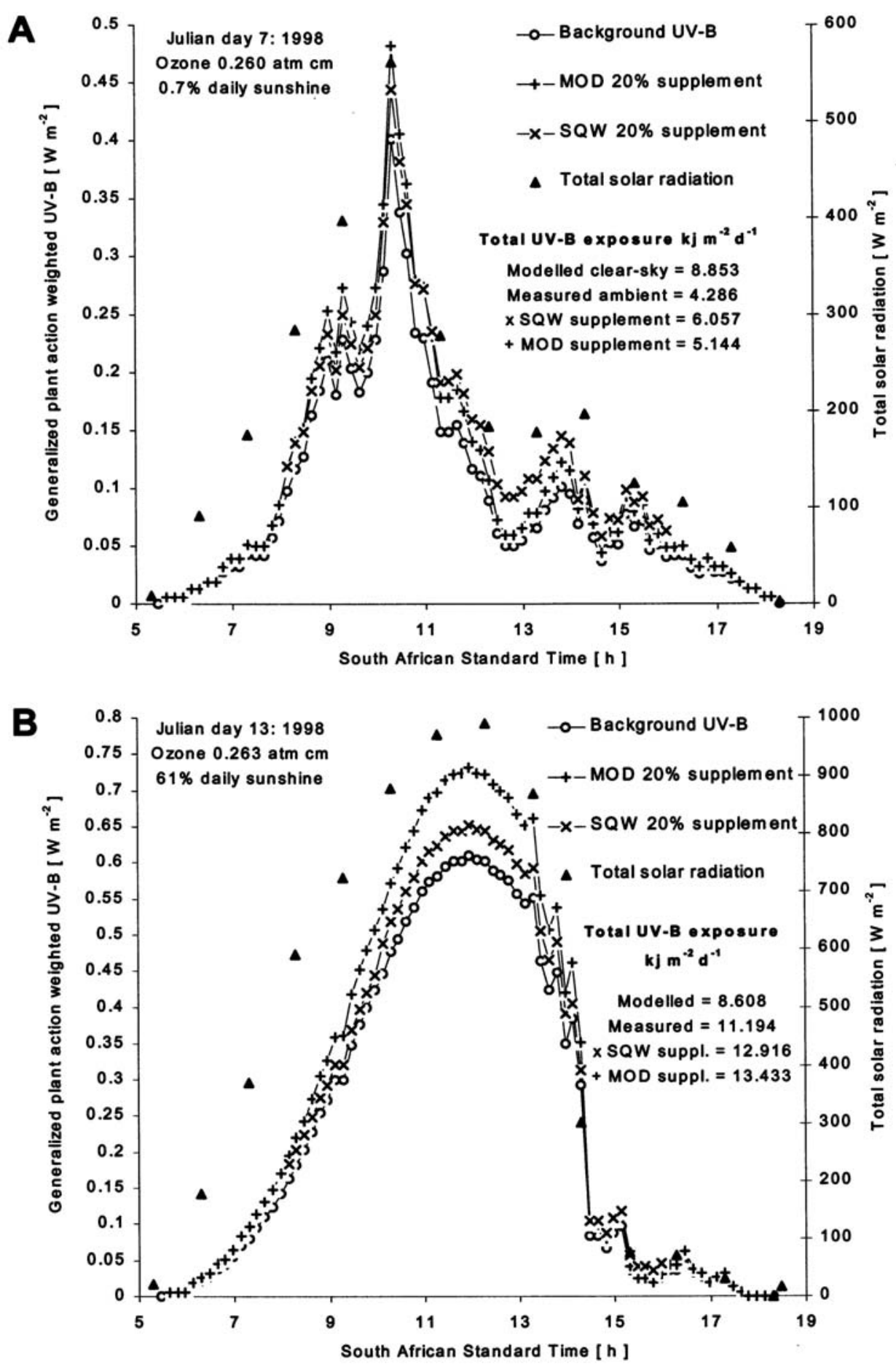

Fig. 1. Instrument measured total solar (300-3000 nm) and biologically weighted UV-B (280-315 nm) radiation, and added UV-B supplements of $20 \%$ above background levels in accordance with step-wise SQW and MOD delivery principles under (A) completely overcast ( $0.7 \%$ daily sunshine duration) and (B) slightly overcast ( $61 \%$ daily sunshine duration) days.

The corresponding total daily UV-B exposure predicted for clear-sky conditions from the computer-encoded semi-empirical model using the total column ozone record of $0.260 \mathrm{~atm} \mathrm{~cm}$ for this specific date was $8.853 \mathrm{~kJ} \mathrm{~m}^{-2} \mathrm{~d}^{-1}$. Addition of $20 \%$ of the 
predicted total daily UV-B exposure, namely $1.771 \mathrm{~kJ} \mathrm{~m}^{-2} \mathrm{~d}^{-1}\left(29.509 \mathrm{~W} \mathrm{~m}^{-2}\right)$, as a supplement to the instrument measured background UV-B irradiances gave a total daily supplemental UV-B exposure in the SQW delivery system of $6.057 \mathrm{~kJ} \mathrm{~m}^{-2} \mathrm{~d}^{-1}$. The corresponding total daily supplemental UV-B exposure rendered in the MOD delivery system on this overcast day was $5.144 \mathrm{~kJ} \mathrm{~m}^{-2} \mathrm{~d}^{-1}$ (Fig. 1(A)).

Total daily supplemental UV-B exposures rendered by step-wise SQW and MOD delivery systems were matched with synchronous measurements of daily sunshine duration expressed as percentages of day length. Exposures were sorted and grouped into 11 incremental sunshine percentage categories in the range $0-100 \%$ daily sunshine duration. Differences between SQW and MOD delivered total daily supplemental UV-B exposures for each sunshine category were calculated as follows:

$$
\% \text { difference }=((\mathrm{SQW}-\mathrm{MOD}) / \mathrm{MOD}) \cdot 100
$$

\subsubsection{Photon flux ratios of ultraviolet-B/total solar radiation}

Instrument measured UV-B irradiances with their added SQW and MOD UV-B supplements were synchronized with independent hourly measurements of TSR (Fig. 1(A) and (B)). Photon flux ratios between SQW delivered UV-B and TSR (SQW/TSR) and MOD delivered UV-B and TSR (MOD/TSR) were computed daily for early morning $(09: 00 \mathrm{~h})$, midday $(12: 00 \mathrm{~h})$ and late afternoon $(15: 00 \mathrm{~h})$ photoperiods. Over an entire year, these computed photon flux ratios included the solar angle range $19-84^{\circ}$, with an average solar angle interval and standard error of $0.009890 .00022^{\circ}$. The computed ratios were sorted and grouped into five incremental solar angle categories, each covering a range of $12^{\circ}$ solar angle, except for the first category which included a range of $19-36^{\circ}$ solar angle. The ratios were matched with synchronous measurements of daily percentage sunshine, and further subdivided into 11 incremental daily percentage sunshine categories. Differences between step-wise SQW and MOD delivered ratios of UV-B/TSR for each solar angle and daily sunshine percentage category were calculated:

$$
\% \text { difference }=((\mathrm{SQW} / \mathrm{TSR}-\mathrm{MOD} / \mathrm{TSR}) / \mathrm{MOD} / \mathrm{TSR}) \cdot 100
$$

\subsection{Statistical analyses}

Differences between step-wise SQW and MOD delivery systems in total daily supplemental UV-B exposures provided and photon flux ratios of UV-B/TSR rendered were tested for normality in distribution. The students t-test was then applied to the normal data to test the null hypotheses that the mean differences in total daily supplemental UV-B exposures and photon flux ratios rendered by the two delivery systems were zero (Bland, 1987). 


\section{Results}

\subsection{Comparison of square-wave and modulated delivery systems}

\subsubsection{Total daily supplemental ultraviolet-B exposures}

Both broad-and narrow-band instrument measurements (Table 1) confirmed that our step-wise SQW delivery system rendered significantly (P 00.01 ) larger total daily supplemental UV-B exposures than a MOD delivery system on only substantially overcast days ( $25 \%$ or less daily sunshine duration). These larger supplemental UV-B

Table 1 Differences (\%) between SQW and MOD delivered total daily supplemental UV-B exposures for added UV-B supplements of three different magnitudes measured by broad-and narrow-band instrumentation

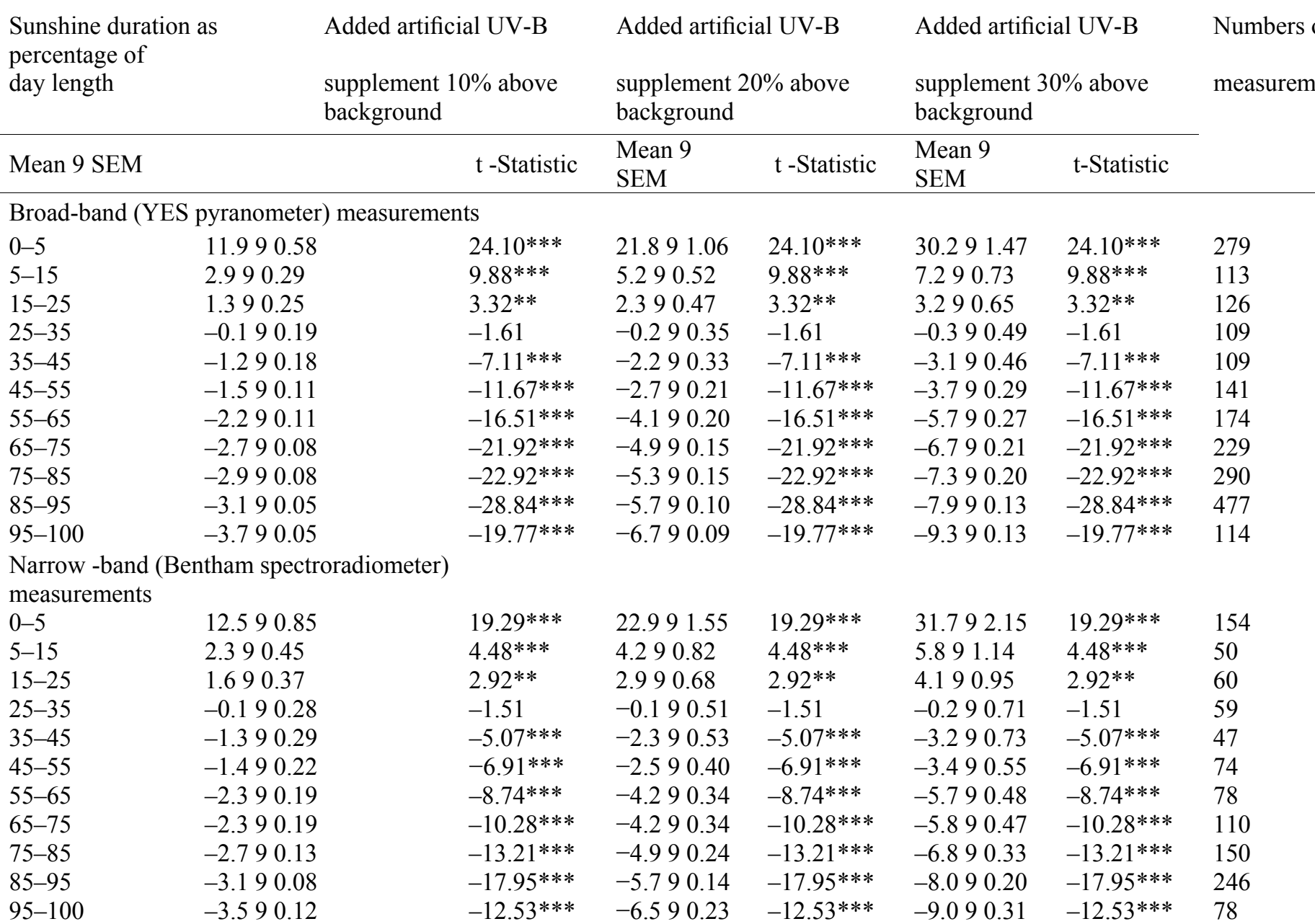

** Statistically significant differences between SQW and MOD delivered exposures at $\mathrm{P}<0.01$.

*** Statistically significant differences between SQW and MOD delivered exposures at $\mathrm{P}<0.001$.

exposures were augmented with increased magnitude of the added artificial UV-B supplement (Fig. 2(A)). However, their ranges did not exceed those in a MOD delivery system by more than $10 \%$ for the added UV-B supplements of realistic magnitude $(30 \%$ 
or less above background), except on virtually completely overcast days (5\% or less daily sunshine duration).
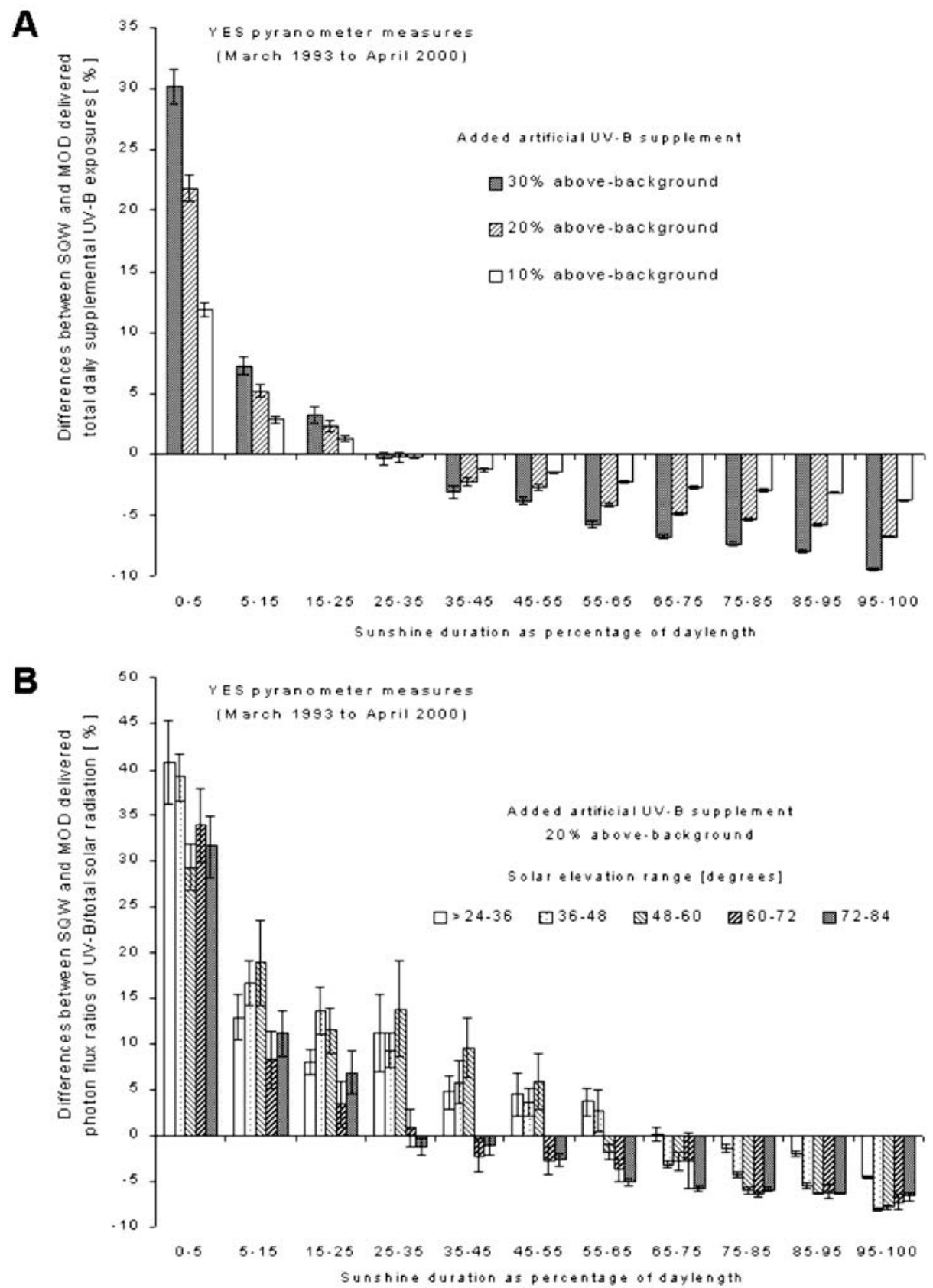

Fig. 2. Differences (\%) between SQW and MOD delivery systems in (A) total daily supplemental UV-B exposures provided and (B) photon flux ratios of UV-B/TSR rendered for 11 categories of daily sunshine duration, five solar angle classes and added UV-B supplements of three different magnitudes.

\subsubsection{Photon flux ratios of ultraviolet-B/total solar radiation}

Our step-wise SQW delivery system also rendered significantly $(P<0.05)$ higher photon flux ratios of UV-B/TSR than a MOD delivery system on only substantially overcast days, the ranges of which were also augmented with increased magnitude of the added artificial UV-B supplement (Table 2). However, these features were restricted to 
high (above $72^{\circ}$ ) solar angles, since with reduced solar angle these significantly $(P 0$ 0.05 ) higher photon flux ratios also included progressively less overcast days (Fig. 2(B)), i.e. days with $45 \%$ or less sunshine duration at solar angles below $36^{\circ}$ (Table 2 ).

\section{Discussion}

The impression created in most criticisms of SQW delivery systems is one of substantial UV-B supplementation excesses, and exaggerated plant responses to the greater total amounts of UV-B supplied (Sullivan et al., 1994; Allen et al., 1998). However, their exist few factual studies of SQW and MOD delivery systems operating concurrently. One published study claimed an up to $30 \%$ greater total daily supplemental UV-B exposure rendered in a SQW than MOD delivery system, and an observed trend towards a lower, though statistically insignificant, photosynthetic capacity in SQW irradiated plants (Sullivan et al., 1994). Noteworthy was that this study's observations were based on extreme experimental protocols. The added artificial UV-B supplements were unrealistically high (60\% above background), and the SQW delivery systems were operated under all climatic conditions, despite some unspecified adjustments to lamp outputs to compensate for overcast days. Our observations indicate much smaller UV-B supplementation excesses in a step-wise SQW delivery system than alleged. Indeed, their ranges did not exceed those in a MOD delivery system by more than $10 \%$ for the added artificial UV-B supplements of realistic magnitude, except on virtually completely overcast days ( $5 \%$ or less sunshine duration) under which lamp banks would normally be switched off. Even switching off of lamp banks on just substantially overcast days (25\% or less daily sunshine duration) resulted in actual inadequate total daily supplemental UV-B exposures rendered in our step-wise SQW delivery system. Furthermore, arguments that SQW delivery systems add a flat irradiance whereas MOD delivery systems follow the peaks in natural sunlight were not supported by our measurements. These clearly indicated that the supplemental UV-B irradiances rendered by both SQW and MOD delivery systems in an outdoor situation closely followed the measured peaks in natural sunlight. However, the SQW delivery system rendered smaller UV-B supplements than the MOD system in the sunlight peaks on both completely and slightly overcast days (Fig. 1(A) and (B)), and vice-versa in the sunlight depressions, indicating that the accumulated effects on plants might not be the same. These UV-B supplementation deficiencies in the SQW delivery system were due to a consistent underestimation of clear-sky background UV-B irradiance by the computer-encoded semiempirical model. Published spectral data along a latitudinal gradient (Caldwell et al., 1980), and one year's spectroradiometer measurements at a separate southern-hemisphere location (Musil et al., 1999) concur with this observation.

It has been proposed that elevated photon flux ratios of UV-B/PAR and UV-B/UV-A are also an important cause of discrepancies between results of field-based SQW and MOD delivery systems (Allen et al., 1999). Increased sensitivity of plants to UV-B radiation at low PAR and UV-A levels have been widely reported (Allen et al., 1998). Studies of Antarctic phytoplankton populations (Smith et al., 1992) have demonstrated photosynthetic inhibition proportional to an increase in the ratio of UV-B/ (UV-A + PAR), but among terrestrial plants there appears to be a threshold of PAR above which 
detrimental UV-B effects are substantially reduced or even eliminated. Indeed, Caldwell et al. (1994) found that in soybean plants exposed to MOD UV-B supplements in the field that significant reductions in biomass only occurred when PAR and UV-A levels are reduced to $50 \%$ their flux in sunlight (161and $1900 \mathrm{mmol} \mathrm{m}^{-2} \mathrm{~s}^{-1} \mathrm{UV}-\mathrm{A}$ and PAR, respectively). However, under these reduced UV-A and PAR levels, no further growth inhibition was observed in experiments where ratios of UV-B/(UV-A + PAR) were increased by 33\%. These findings are supported by those of Deckmyn and Impens (1997) who found that a $30 \%$ increase in UV-B irradiance caused equivalent reductions in total dry weight and photosynthesis of rye plants at four incremental PAR levels below 1100

Table 2 Differences (\%) between SQW and MOD delivered photon flux ratios of UV-B/TSR for three solar angle classes and added UV-B supplements of three different magnitudes

$\begin{array}{llll}\text { Sunshine duration as percentage of } & \text { Added artificial UV-B } & \text { Added artificial UV-B } & \text { Added artificial UV }\end{array}$

day length $\quad$ supplement $10 \%$ above

supplement $20 \%$ above

supplement $30 \%$ above

background

background

\begin{tabular}{|c|c|c|c|c|c|c|c|}
\hline & Mean 9 SEM & $t$-Statistic & Mean 9 SEM & $t$-Statistic & Mean 9 SEM & $t$-Statistic & \\
\hline \multicolumn{8}{|c|}{ Solar angle range: $124^{\circ}-36^{\circ}$} \\
\hline $0-5$ & 22.292 .49 & $8.80 * * *$ & 40.894 .56 & $8.79 * * *$ & 56.496 .32 & $8.80 * * *$ & 138 \\
\hline $5-15$ & 7.191 .36 & $2.74 * *$ & 13.092 .49 & $2.74 * *$ & 17.993 .45 & $2.74 * *$ & 76 \\
\hline $15-25$ & 4.490 .75 & $4.76^{* *}$ & 8.091 .37 & $4.76^{* *}$ & 11.191 .90 & $4.75^{* *}$ & 76 \\
\hline $25-35$ & 6.192 .31 & $2.26^{*}$ & 11.294 .24 & $2.26^{*}$ & 15.595 .88 & $2.26^{*}$ & 54 \\
\hline $35-45$ & 2.691 .00 & $2.03^{*}$ & 4.791 .84 & $2.04 *$ & 6.592 .55 & $2.04 *$ & 75 \\
\hline $45-55$ & 2.491 .24 & 1.36 & 4.592 .28 & 1.36 & 6.293 .16 & 1.35 & 73 \\
\hline $55-65$ & 2.090 .85 & 1.56 & 3.791 .55 & 1.56 & 5.192 .15 & 1.56 & 130 \\
\hline $65-75$ & 0.090 .38 & -1.07 & 0.190 .70 & -1.08 & 0.190 .97 & -1.08 & 198 \\
\hline $75-85$ & -0.890 .25 & -0.74 & -1.490 .45 & -0.75 & -1.990 .62 & -0.75 & 304 \\
\hline $85-95$ & -1.190 .16 & $-5.16 * * *$ & -2.090 .29 & $-5.18 * * *$ & -2.890 .40 & $-5.17 * * *$ & 656 \\
\hline $95-100$ & -2.590 .06 & $-6.50 * * *$ & -4.690 .12 & $-6.50 * * *$ & -6.390 .16 & $-6.51 * * *$ & 210 \\
\hline \multicolumn{8}{|c|}{ Solar angle range: $48^{\circ}-60^{\circ}$} \\
\hline $0-5$ & 16.091 .37 & $12.55^{* * *}$ & 29.392 .51 & $12.55 * * *$ & 40.693 .48 & $12.55 * * *$ & 185 \\
\hline $5-15$ & 10.392 .54 & $4.54 * * *$ & 18.894 .66 & $4.55 * * *$ & 26.196 .45 & $4.55^{* * *}$ & 79 \\
\hline $15-25$ & 6.291 .38 & $4.18 * * *$ & 11.492 .53 & $4.19 * * *$ & 15.893 .50 & $4.19 * * *$ & 75 \\
\hline $25-35$ & 7.592 .86 & $2.25^{*}$ & 13.895 .24 & $2.25^{*}$ & 19.197 .26 & $2.25 *$ & 64 \\
\hline $35-45$ & 5.291 .77 & $2.12 *$ & 9.693 .25 & $2.12^{*}$ & 13.394 .50 & $2.12 *$ & 80 \\
\hline $45-55$ & 3.291 .69 & 1.12 & 5.993 .10 & 1.11 & 8.294 .29 & 1.11 & 94 \\
\hline $55-65$ & -1.090 .48 & $-3.46^{* * *}$ & -1.890 .88 & $-3.46 * * *$ & -2.591 .22 & $-3.46^{* * *}$ & 112 \\
\hline $65-75$ & -1.690 .55 & $-6.25^{* * *}$ & -2.991 .02 & $-6.26 * * *$ & -4.091 .41 & $-6.26 * * *$ & 116 \\
\hline $75-85$ & -3.390 .17 & $-8.30 * * *$ & -6.090 .30 & $-8.30 * * *$ & -8.390 .42 & $-8.29 * * *$ & 147 \\
\hline $85-95$ & -3.590 .11 & $-8.30 * * *$ & -6.390 .20 & $-8.31 * * *$ & -8.890 .28 & $-8.30 * * *$ & 133 \\
\hline $95-100$ & -4.390 .12 & $-9.27 * * *$ & -7.990 .23 & $-9.28 * * *$ & -10.990 .32 & $-9.29 * * *$ & 33 \\
\hline \multicolumn{8}{|c|}{ Solar angle range: $72^{\circ}-84^{\circ}$} \\
\hline $0-5$ & 17.291 .79 & $10.83 * * *$ & 31.693 .27 & $10.83 * * *$ & 43.794 .53 & $10.83 * * *$ & 122 \\
\hline $5-15$ & 6.191 .40 & $3.64 * * *$ & 11.292 .57 & $3.64 * * *$ & 15.493 .56 & $3.64 * * *$ & 50 \\
\hline $15-25$ & 3.791 .30 & $2.45^{*}$ & 6.892 .39 & $2.45^{*}$ & 9.593 .30 & $2.45^{*}$ & 54 \\
\hline $25-35$ & -0.790 .49 & $-2.81 *$ & -1.390 .90 & $-2.80 *$ & -1.891 .25 & $-2.81 *$ & 55 \\
\hline $35-45$ & -0.690 .58 & $-2.86 * * *$ & -1.191 .07 & $-2.85^{*}$ & -1.591 .48 & $-2.85^{*}$ & 52 \\
\hline $45-55$ & -1.590 .41 & $-6.31 * * *$ & -2.790 .76 & $-6.32 * * *$ & -3.891 .05 & $-6.32 * * *$ & 68 \\
\hline $55-65$ & -2.890 .19 & $-8.26^{* * *}$ & -5.190 .35 & $-8.25 * * *$ & -7.090 .49 & $-8.25 * * *$ & 73 \\
\hline $65-75$ & -3.190 .17 & $-16.09^{* * *}$ & -5.890 .31 & $-16.08 * * *$ & -8.090 .43 & $-16.08 * * *$ & 71 \\
\hline $75-85$ & -3.290 .15 & $-14.01 * * *$ & -5.890 .27 & $-14.02 * * *$ & -8.190 .37 & $-14.02 * * *$ & 73 \\
\hline $85-95$ & -3.590 .10 & $-10.13 * * *$ & -6.490 .18 & $-10.12 * * *$ & -8.890 .25 & $-10.12 * * *$ & 66 \\
\hline $95-100$ & -3.790 .26 & $-14.36^{* * *}$ & -6.790 .48 & $-14.20 * * *$ & $\begin{array}{l}-9.390 .67 \\
\end{array}$ & $-14.16^{* * *}$ & 5 \\
\hline
\end{tabular}

* Statistically significant differences between SQW and MOD delivered exposures at $P 00.05$

** Statistically significant differences between SQW and MOD delivered exposures at P00.01.

*** Statistically significant differences between SQW and MOD delivered exposures at P00.001. 
$\mathrm{mmol}^{-2} \mathrm{~s}^{-1}$. Our observations, however, show that ranges of photon flux ratio increases in our stepwise SQW delivery system were well below reported thresholds inhibiting to plant growth at all solar angles for the added UV-B supplements of realistic magnitude, except on virtually completely overcast days. Nevertheless, there is some experimental evidence suggesting that smaller, though unspecified, changes in photon flux ratios, especially at low PAR levels during early or late daily photoperiods, may impact on plant performance. Sullivan et al. (1994), for example, reported that slight increases in UV-B radiation during early morning, and more importantly during late evening photoperiods in a MOD but not SQW delivery system differentially affected plant carbohydrate metabolism. Plants in the MOD delivery system displayed significantly lower starch levels at the end of the night in spite of a higher daily starch accumulation rate, which pointed to an altered day length response to photosynthate partitioning. Perhaps, some developmental and biochemical responses may be affected by differential timing of UVB exposures in SQW and MOD delivery systems, but their relevance have yet to be substantiated. Indeed, these are controversial considering the general insensitivity of many plants to realistic UV-B increases in context of their normal radiation environment, and the extreme measurements that often have to be employed to elicit significant UV-B effects in plants (Fiscus and Booker, 1995). Clearly, MOD delivery systems do provide more realistic assessments of UV-B increases and their application should be encouraged where economically feasible. However, likewise there seems little justification on balance for branding results derived from all field-based SQW delivery systems as exaggerated where sensible irradiation protocols and realistic UV-B supplements have been applied. Indeed, the results of this study provide a means of gauging the magnitude of UV-B supplementation inaccuracies in a typical out-door step-wise SQW supplementation system using a commonly monitored meteorological criterion, daily sunshine duration, as a reference. Utilization of authentic ozone data and the computer-encoded semi-empirical model published by Björn and Murphy (1985) are prerequisites in such assessments, and implementation of corrective measurements.

\section{References}

Allen, D.A., Nogues, S., Baker, N.R., 1998. Ozone depletion and increased UV-B radiation: is there a real threat to photosynthesis. J. Exp. Bot. 49, 1775-1788.

Allen, D.A., Nogues, S., Morison, J.I.L., Greenslade, P.D., McLeod, A.R., Baker, N.R., 1999. A thirty percent increase in UV-B has no impact on photosynthesis in well-watered and droughted pea plants in the field. Global Change Biol. 5, 235-244.

Björn, L.O., Murphy, T.M., 1985. Computer calculations of solar ultraviolet radiation at ground level. Physiol. Veg. 23, 555-561.

Bland, M., 1987. Introduction to Medical Statistics. New York University Press, New York.

Bodeker, G.E., McKenzie, R.L., 1996. An algorithm for inferring surface UV irradiance including cloud effects. J. Appl. Meteorol. 35, $1860-1877$.

Booker, F.L., Fiscus, R.E., Philbeck, A.S., Miller, J.E., Heck, W.W., 1992. A supplemental ultraviolet-B radiation system for open-top field chambers. J. Environ. Qual. 21, 56-61.

Burrows, J.P., Weber, M., Buchwitz, M., Rozanov, V., Lad-statter-Weissenmayer, A., Richter, A., DeBeek, R., Hoo-gen, R., Bramstedt, K., Eichmann, K.-U., Eisinger, M., 1999. The Global Ozone Monitoring Experiment (GOME): mission concept and first scientific results. J. Atmos. Sci. 56, 151-175.

Caldwell, M.M., 1971. Solar ultraviolet radiation and the growth and development of higher plants. In: Giese, A.C. (Ed.), Photophysiology, vol. 6. Academic Press, New York, pp. 131-177.

Caldwell, M.M., Robberecht, R., Billings, W.D., 1980. A steep latitudinal gradient of solar ultraviolet-B radiation in the arctic-alpine life zone. Ecology 61, 600-611.

Caldwell, M.M., Gold, W.G., Harris, G., Ashurst, C.W., 1983. A modulated lamp system for solar UV-B (280-320 nm) supplementation studies in the field. Photochem. Photobiol. 37, 479-485.

Caldwell, M.M., Camp, L.B., Warner, C.W., Flint, S.D., 1986. Action spectra and their key role in assessing biological consequences of solar UV-B change. In: Worrest, R.C., Caldwell, M.M. (Eds.), Stratospheric Ozone Reduction, Solar Ultraviolet Radiation and Plant Life. Springer, Berlin, pp. 87-111.

Caldwell, M.M., Flint, S.D., Searles, P.S., 1994. Spectral balance and UV-B sensitivity of soybean: a field experiment. Plant Cell Environ. 17, 267-276.

Caldwell, M.M., Teramura, A.H., Tevini, M., Bornmann, L.O., Kulandaivelu, G., 1995. Effects of solar ultraviolet radiation on 
terrestrial plants. Ambio 24, 166-173.

Climate of South Africa, 1986. Climatic Statistics up to 1984. Weather Bureau, Department of Environment Affairs, 40. Government Printer, Pretoria.

Coohill, T.P., 1991. Action spectra again? Photochem. Photobiol. 54, 859-870.

Coohill, T.P., 1992. Action spectra revisited. Photochem. Photobiol. B Biol. 13, 95-100.

Deckmyn, G., Impens, I., 1997. The ratio of UV-B/photosyn-thetically active radiation determines the sensitivity of rye to increased UV-B radiation. Environ. Exp. Bot. 37, 3-12.

Döhring, T., Kofferlein, M., Thiel, S., Seidlitz, H.K., 1996. Spectral shaping of artificial UV-B irradiation for vegetation stress research. J. Plant Physiol. 148, 115-119.

Fiscus, E.L., Booker, F.L., 1995. Is increased UV-B a threat to crop photosynthesis and productivity? Photosynth. Res. 43, 81-92.

Flint, S.D., Caldwell, M.M., 1996. Scaling plant ultraviolet spectral responses from laboratory action spectra to field spectral weighting factors. J. Plant Physiol. 148, 107-114.

Fraser, P.J., Prather, M.J., 1999. Uncertain road to ozone recovery. Nature 398, 663-664.

Frederick, J.E., Steele, D., 1995. The transmission of sunlight through cloudy skies: an analysis based on standard meteorological information. J. Appl. Meteorol. 34, 2755-2761.

Green, A.E.S., 1983. The penetration of ultraviolet radiation to the ground. Physiol. Plant 58, 351-359.

Green, A.E.S., Sawada, T., Shettle, E.P., 1974. The middle ultraviolet reaching the ground. Photochem. Photobiol. 19, $251-259$.

Green, A.E.S., Cross, K.R., Smith, L.A., 1980. Improved analytical characterization of skylight. Photochem. Photobiol. 31, 59-65.

http://toms.gsfc.nasa.gov/

Herman, J.R., Bhartia, P.K., Krueger, A.J., McPeters, R.D., Wellemeyer, C.G., Seftor, C.J., Jaross, G., Schlesinger, B.M., Torres, O., Labow, G., Byerly, W., Taylor, S.L., Swissler, T., Cebula, R.P., 1996. Meteor-3 Total Ozone Mapping Spectrometer (TOMS) Data Protocols User's Guide, NASA Ref. Publ. No. 1393 (PDF file available on ).

Jansen, M.A.K., Gaba, V., Greenberg, B.M., 1998. Higher plants and UV-B radiation: balancing damage, repair and acclimation. Trends Plant Sci. 3, 131-135.

http://toms.gsfc.nasa.gov/Krueger, A.J., Bhartia, P.K., McPeters, R.D., Herman, J.R., Wellemeyer, C.G., Jaross, G., Seftor, C.J., Torres, O., Labow, G., Byerly, W., Taylor, S.L., Swissler, T., Cebula, R.P., 1998. ADEOS Total Ozone Mapping Spectrometer (TOMS) Data Products User's Guide, NASA Ref. Publ. (PDF file available on ).

Laakso, K., Huttunen, S., 1998. Effects of the ultraviolet-B radiation (UV-B) on conifers: a review. Environ. Pollut. 99, $319-328$.

McKinley, A.F., Diffey, B.L., 1987. A reference action spectrum for ultraviolet induced erythema in human skin. In: Passchier, W.R., Bosnjakovic, B.F.M. (Eds.), Human Exposure to Ultraviolet Radiation: Risks and Regulations. Elsevier, Amsterdam.

McLeod, A.R., 1997. Outdoor supplementation systems for studies of the effects of increased UV-B radiation. In: Rozema, J., Gieskes, W.W.C., Greijn, S.C., van de Nolan, C., Boois de, H. (Eds.), UV-B and Biosphere. Kluwer, Dordrecht, pp. 78-92.

http://toms.gsfc.nasa.gov/McPeters, R.D., Bhartia, P.K., Krueger, A.J., Herman, J.R., Schlesinger, B.M., Wellemeyer, C.G., Seftor, C.J., Jaross, G., Taylor, S.L., Swissler, T., Torres, O., Labow, G., Byerly, W., Cebula, R.P., 1996. Nimbus-7 Data Products User's Guide, NASA Ref. Pub. No. 1384 (PDF file available on ).

McPeters, R.D., Bhartia, P.K., Krueger, A.J., Herman, J.R., Wellemeyer, C.G., Seftor, C.J., Jaross, G., Torres, O., Moy, L., Labow, G., Byerly, W., Taylor, S.L., Swissler, T., Cebula, R.P., 1998. Earth Probe Total Ozone Mapping Spectrometer (TOMS) Data Products User's Guide, NASA Ref. Pub. (PDF file available on http:// toms.gsfc.nasa.gov/).

Middleton, E.M., Teramura, A.H., 1993. Potential errors in the use of cellulose diacetate and mylar filters in UV-B radiation studies. Photochem. Photobiol. 57, 744-751.

Musil, C.F., Midgely, G.F., Wand, S.J.E., 1999. Carryover of enhanced UV-B exposure effects to successive generations of a desert annual: interaction with atmospheric $\mathrm{CO}_{2}$ and nutrient supply. Global Change Biol. 5, 311-332.

Newsham, K.K., McLeod, A.R., Greenslade, P.D., Emmett, B.A., 1996. Appropriate controls in outdoor UV-B supplementation experiments. Global Change Biol. 2, 319-324.

Nunez, M., Forgan, B., Roy, C., 1994. Estimating ultraviolet radiation ate the earth's surface. Int. J. Biometeorol. $38,5-17$.

Rozema, J., van de Staaij, J., Björn, L.O., Caldwell, M., 1997. UV-B as an environmental factor in plant life: stress and regulation. Trends Ecol. Evol. 12, 22-28.

Rozema, J., van de Staaij, J., Björn, L.O., De Bakker, N., 1999. Depletion of stratospheric ozone and solar UV-B radiation: evolution of land plants, UV-screens and functions of polyphenolics. In: Rozema, J. (Ed.), Stratospheric Ozone Depletion: The Effects of Enhanced UV-B Radiation on Terrestrial Ecosystems. Backhuys Publishers, Leiden, The Netherlands, pp. 1-19.

Rundel, R.D., 1983. Action spectra and estimation of biologically effective UV radiation. Physiol. Plant. 58, $360-366$.

Rundel, R.D., 1986. Computation of spectral distribution and intensity of solar UV-B radiation. In: Worrest, R.C., Caldwell, M.M. (Eds.), Stratospheric Ozone Reduction, Solar Ultraviolet Radiation and Plant Life. Springer, Berlin, p. 49.

Smith, $\quad$ R.C., Prezelin, B.B., Baker, K.S., Bidigare, R.R., Boucher, N.P., Coley, T., Karentz, D., MacIntyre, S., Matlick, H.A., Menzies, D., Ondrusek, M., Wan, Z., Walters, K.J., 1992. Ozone depletion: ultraviolet radiation and phytoplankton biology in Antarctic waters. Science 255, 952-959.

Sullivan, J.H., Teramura, A.H., Adamse, P., Kramer, G.F., Upadhyaya, A., Britz, S.J., Krizek, D., Mirecki, R., 1994. Comparison of the response of soybean to supplemental UV-B radiation supplied by either square-wave or modulated irradiation systems. In: Biggs, R.H., Joyner, M.E.B. (Eds.), Stratospheric Ozone Depletion: UV-B radiation in the Biosphere. NATO ASI Series I, vol. 18. Springer, Berlin-Heidelberg, pp. 211-220.

Teramura, A.H., 1983. Effects of ultraviolet UV-B radiation on growth and yield of crop plants. Physiol. Plant 58, $415-427$.

Thiel, S., Steiner, K., Seidlitz, H.K., 1997. Modification of global erythemally effective irradiance by clouds. Photochem. Photobiol. $65,969-973$. 